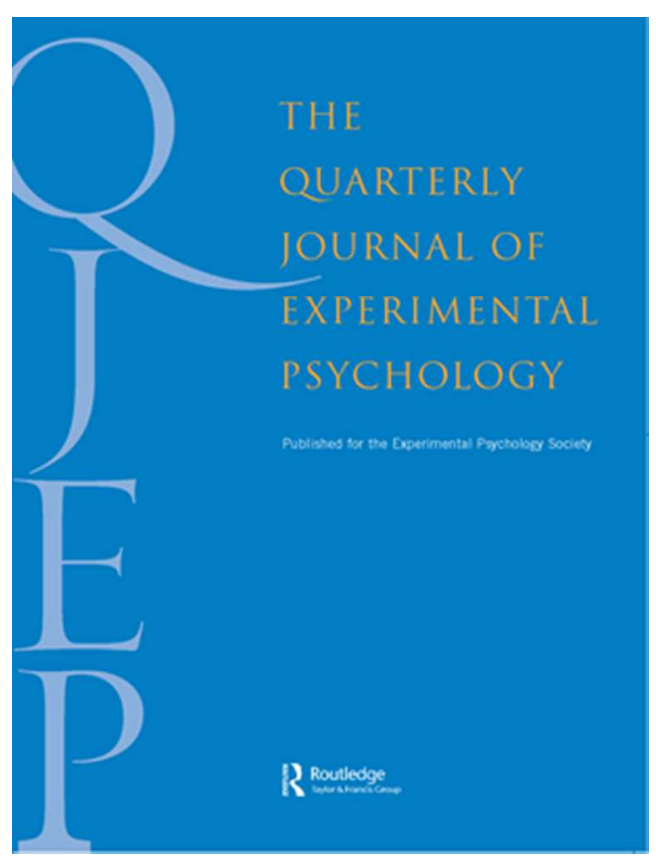

\title{
Optimal Time Discrimination
}

\begin{tabular}{|r|l|}
\hline Journal: & Quarterly Journal of Experimental Psychology \\
\hline Manuscript ID: & QJE-STD 14-039.R1 \\
\hline Manuscript Type: & Standard Article \\
\hline Date Submitted by the Author: & n/a \\
\hline Complete List of Authors: & $\begin{array}{l}\text { Çoskun, Filiz; Koç University, Psychology } \\
\text { Sayalı Ungerer, Zeynep; Koç University, Psychology } \\
\text { Gürbüz, Emine; Koç University, Psychology } \\
\text { Balcı, Fuat; Koç University, Psychology }\end{array}$ \\
\hline Keywords: & $\begin{array}{l}\text { Decision making, Interval Timing, Optimality, Response Times, Temporal } \\
\text { Bisection }\end{array}$ \\
\hline &
\end{tabular}

SCHOLARONE $^{\text {Im }}$

Manuscripts 


\section{OPTIMAL TIME DISCRIMINATION}

Running Head: Optimal Time Discrimination

Corresponding Author:

Fuat Balc1, PhD.

Assistant Professor

Department of Psychology

Koç University

Rumeli Feneri Yolu, Sariyer 34450

Istanbul, Turkey

Tel: +90 (212) 3381138

Fax: +90 (212) 3381415

Email: fbalci@ku.edu.tr 


\begin{abstract}
In the temporal bisection task, participants categorize experienced stimulus durations as short or long based on their similarity to previously acquired short and long reference durations. Reward maximization in this task requires integrating endogenous timing uncertainty as well as exogenous probabilities of the reference durations into temporal judgments. We conducted two experiments testing human participants on the temporal bisection task with different short and long reference duration probabilities (exogenous probability) in two separate test sessions for each participant. Incorrect categorizations were not penalized in Experiment 1 whereas they were penalized in Experiment 2 leading to different levels of stringency in the reward functions that participants tried to maximize. We evaluated the observed temporal judgments within the framework of optimality. Our participants adapted their choice behavior in a nearly optimal fashion and earned nearly the maximum possible expected gain they could attain given their level of endogenous timing uncertainty and exogenous reference duration probabilities in both experiments. These results pointed at the optimality of human temporal risk-assessment in the temporal bisection task. The analysis of categorization response times (RTs) further revealed that long categorization RTs were overall faster than short categorization RTs and that short but not long categorization RTs were modulated by reference duration probability manipulations. These observations suggested an asymmetry between short and long categorizations in the temporal bisection task.
\end{abstract}

Keywords: Decision making, Interval timing, Optimality, Response times, Temporal bisection 


\section{INTRODUCTION}

Time is an inherent feature of many simple decisions that humans and non-human animals routinely make. Despite the apparent simplicity of many time-based decisions, due to our imprecise time-keeping ability as manifested in trial-to-trial variability in timed responses, every temporal decision we make is subject to our own endogenous timing uncertainty. Thus, even when they are about temporally deterministic scenarios, time-based decisions require temporal risk assessment. Outside the lab, this ubiquitous source of uncertainty is often accompanied by exogenous probabilistic relations. In these cases, reward maximization entails an accurate representation of both one's own endogenous timing uncertainty and exogenous probabilities, and their normative integration into decision-making. We investigated the temporal discrimination performance of human participants under different exogenous probability conditions with both lenient (due to lack of penalty for errors) and stringent (due to penalty for errors) reward functions. Observed temporal choice behaviors were evaluated within the framework of optimality defined based on participants' endogenous timing uncertainty (estimated from trial-to-trial variability in timing behavior) as well as experimentally manipulated exogenous probabilities.

Evolution appears to have favored effectively a flexible stopwatch-like mechanism that enables many organisms to time intervals from seconds to minutes (Buhusi \& Meck, 2005). This ability helps animals to cluster their activities around biologically important time intervals (Drew, Zupan, Cooke, Couvillon \& Balsam, 2005), calculate reward rates (Gallistel et al., 2007), prefer the sooner of the two rewards with equal magnitudes (Gibbon \& Church, 1981), and make other biologically critical time- 
related decisions (Kacelnik \& Brunner, 2002). The statistical properties of interval timing are important for understanding the role of endogenous timing uncertainty in determining the optimal temporal decision strategies. To this end, timed responses are approximately Gaussian distributed with a high degree of accuracy (e.g., timed response distributions are centered on the corresponding target times) but limited precision (timed responses exhibit substantial trial-to-trial variability). Within an individual, this temporal imprecision has a well-defined relationship to the timed intervals: the standard deviation of the time reproductions is proportional to their mean (i.e., constant coefficient of variation (CV)), which defines the scalar property of interval timing (Gibbon, 1977). Consequently, many species capture and exploit the environmental statistics under similar precision constraints that relate to their time representations.

The interval timing literature has traditionally focused on exploring the psychophysical properties of timing ability. Relatively few number studies have incorporated the psychophysics of interval timing into the study of the role of interval timing in reward maximization. In one of these studies, Balc1, Freestone and Gallistel (2009) tested both humans and mice on the switch task (Balc1 et al, 2008; Killeen \& Fetterman, 1988; Platt \& Davis, 1983). The switch task is a temporal discrimination procedure, where subjects initiate responses at the location associated with the short delay to reinforcement, and in long trials switch to the location associated with long delay to reinforcement when responses at the former location were not reinforced after the short delay. When subjects switch early in short trials (switch $<$ short duration) or do not switch on time in long trials (switch>long duration), they either do not receive a reward or get penalized depending on the pay-off matrix. 
Balc1 et al (2009) manipulated the probability of short and long trials, and formulated that the optimal switch latency depended on both subjects' endogenous timing uncertainty and the relative frequencies of short and long trials. Importantly, both humans and mice maximized their reward in this task, earning nearly the maximum possible expected gain calculated for the level of their endogenous timing uncertainty (i.e., CV of timed responses), experienced exogenous probability (e.g., probability of long trial), and the pay-off matrix (e.g., gains/losses associated with different consequences).

Corroborating this conclusion, recent studies also showed nearly optimal performance of humans in different tasks that specifically required taking account of endogenous timing uncertainty for reward maximization (Balcı et al, 2011a [also in rats], 2011c; Çavdaroğlu, Zeki, \& Balc1, (in press); Hudson, Maloney \& Landy, 2008; Jazayeri \& Shadlen, 2010; Simen, Balc1, deSouza, Cohen \& Holmes, 2011; for review see Balc1 et al, 2011a).

These relatively recent studies however have primarily investigated time-based decisions about events that are likely to happen in the future (prospective), leaving the question of the optimality of temporal decisions about events that have already happened unanswered. Although it is not a purely retrospective decision-making task (should not be confused with retrospective timing), the classical temporal bisection task can partially capture these decisions since participants indicate their decisions following the cessation of the timing signal. In this task, human participants are asked to categorize experienced durations as short or long depending on their similarity to the short and long reference durations. Time intervals that are to-be-judged include the reference durations (i.e., short and long durations) and durations between them (intermediate durations). Intermediate 
durations enable the evaluation of the perceptual aspects of timing as no feedback is provided based on their categorization.

In this study, we aimed to evaluate the optimality of temporal bisection performance of human participants given their endogenous timing uncertainty as well as experienced exogenous probabilities. Endogenous timing uncertainty level of each participant was estimated from his/her temporal judgments. The exogenous probabilities were manipulated by changing the frequency of short and long reference durations within a given participant between two different sessions. We expected the participants to favor long categorizations with increasing probability of long reference duration and short categorizations with decreasing probability of short reference duration (leftward and rightward shift in choice functions, respectively). More importantly, based on earlier studies demonstrating optimal human temporal risk assessment performance, we expected these adaptive biases in temporal choice behavior to occur in an optimal fashion by factoring in the endogenous timing uncertainty and exogenous probabilities. Insensitivity to exogenous probabilities and/or levels of endogenous timing uncertainty on the other hand would lead to deviations from optimality. These questions were investigated in two separate experiments. In Experiment 1 optimality entailed maximizing a liberal reward function with no penalty for incorrect categorizations whereas in Experiment 2 optimality entailed maximizing a more stringent reward function due to the explicit penalty for incorrect categorizations.

The temporal bisection task has both prospective (during stimulus) and retrospective (post-stimulus) decision components. In cases when participants are not allowed to respond prior to the cessation of the timing stimulus (as in our experiment), 
the modulation of post-stimulus short and long categorization RTs as a function of test durations would be informative in terms of the underlying decision processes. Under this rationale, we also evaluated the response time data collected in our experiments. Based on the assumption that participants were also engaged in temporal judgments continuously during the timing stimulus (see Klapproth \& Müller, 1998; Klapproth \& Wearden, 2012), we expected overall faster long categorization RTs compared to short categorization RTs since the participants could commit to long categorization decisions made during stimulus but not to short categorization decisions. Based on this presumed asymmetry between short and long categorizations in terms of decision pre-commitment, we expected short categorization reaction times to be more sensitive to manipulations in exogenous probabilities.

\section{METHODS}

\section{Participants:}

A total of 48 university students served as participants in this study. 24 participants (17 females, 7 males; with an age $M=23.25$ years, $S D=3.8$ ) were tested in Experiment 1 and 24 participants (18 females, 6 males; with an age $M=20.83$ years, $S D=2.48$ ) were tested in Experiment 2. The participants were recruited through a publically available newsletter on the Koç University website. The experiment comprised two 50 minute-long (fixed session duration) daily temporal bisection sessions and one 10 minute-long visual discrimination task after the second temporal bisection session. The participants received monetary reward based on their performance in all three experimental sessions. 


\section{$\underline{\text { Stimuli and apparatus: }}$}

In the temporal bisection task, the timing stimuli included a square $(40 \times 40$ pixels $)$

presented in the middle of the screen with a black background. The period for which the square was visible on the screen was the timing signal. All sessions had fixed durations and the participants could take a break in between the test blocks. The display was generated in MATLAB on a Macintosh computer, using the Psychophysics Toolbox extension (Kleiner, Brainard \& Pelli, 2007). The responses were collected with a computer keyboard.

\section{Procedure:}

1. Temporal Bisection Task:

Familiarization: The participants were presented with the two reference durations (1000 ms vs. $1500 \mathrm{~ms}$ ) four times in an alternating order followed by text indicating whether it was a short or long reference.

Practice: Short and long durations were presented in a pseudo-random order. Participants categorized these durations as short or long by pressing the "V" key with their left and the "N" key with their right index finger, respectively. The practice phase continued for at least 30 trials and until the discrimination accuracy in the last 25 trials was at least $90 \%$. The participants received feedback about the accuracy of their categorizations. For correct categorizations, a brief beep sound was played and the text "Correct" was presented in green for $750 \mathrm{~ms}$ at the top of the screen. For errors, a brief buzzer sound was played, and the text "Incorrect" was presented in red for $750 \mathrm{~ms}$ at the 
top of the screen. The participants were asked not to respond prior to the cessation of the stimulus that signaled the time interval to-be-judged. In case of key presses during the stimulus (premature) or wrong key presses (keys other than "V" or "N"), a warning phrase was presented in purple for $1250 \mathrm{~ms}$. For each condition, probabilities of short and long reference durations were manipulated (see Test). The participants did not accrue any points during the practice phase.

Test:

Five different conditions were defined based on different reference duration probabilities $\left(p\left(T_{s}\right) /\left[p\left(T_{s}\right)+p\left(T_{L}\right)\right]=.10, .25, .50, .75\right.$, or $.90 ;$ referred to as $\left.p\left(T_{s}\right)\right)$. Note that sum of $p\left(T_{s}\right)+p\left(T_{L}\right)$ was always .36 (see below for details). Each participant was tested on one of the four unequal probability conditions in one session and the equal probability condition in the other (order counter-balanced). The participants were informed that, unlike the practice phase, in the test phase intermediate durations would be presented intermixed with the reference durations and that they should categorize each duration as short or long (Figure 1). Each set contained nine durations (including the reference durations), spaced at logarithmically equal distances. Intermediate durations were 1052, 1107, 1164, $1225,1288,1355$, and $1426 \mathrm{~ms}$ and constituted $64 \%$ of the trials; with an average of 424 intermediate duration trials per participant for each session (Experiment 1 and 2). The participants were only rewarded for their correct categorizations of the reference durations in both experiments. In Experiment 1 the participants were not penalized for their incorrect categorizations of the reference durations (only feedback was provided) whereas in Experiment 2 the participants were penalized for their incorrect 
categorizations $(-2 \times$ Reward). Neither feedback nor reward was provided for the categorization of the intermediate durations in order to capture the purely perceptual aspects of temporal discrimination (note that there is no absolute correct categorization for an intermediate duration). The participants were asked to respond as accurately and quickly as possible. This instruction was provided particularly because the response times constituted a unit of analysis in this study. Since the participants could not respond during the timing stimulus, the response time (RT) measurements started at the end of the stimulus that signaled the to-be-judged duration. The participants could take a break and restart testing after every $10^{\text {th }}$ trial (except during the first 30 practice trials).

\section{[FIGURE 1 ABOUT HERE]}

Two-back Working Memory Secondary Task: The participants were instructed not to count or engage in any rhythmic activity such as tapping. In order to prevent chronometric counting, the participants were tested on a concurrent two-back working memory task. After each categorization response, a number selected randomly from 1 to 9 was presented in the middle of the screen for $750 \mathrm{~ms}$. The participants were told that after some trials they would be asked to recognize the number they had seen two trials ago. The number of trials between working memory "interrogations" was sampled from a truncated Poisson distribution (lower bound of 3 and upper bound of 30) with a mean of 10. In the interrogation trials, a number (with $p($ target $)=.50)$ was presented and the participants were asked to press "V" if it was the number they saw two trials back and 
"N" if not. The participants received feedback regarding the accuracy of their working memory performance (for $1000 \mathrm{~ms}$ ).

The inter-trial intervals were sampled from a truncated exponential with a mean of $500 \mathrm{~ms}$ and an upper bound of $1 \mathrm{~s}$. At the beginning of the experiment, the participants were told that their earnings from the timing trials would be weighted by the proportion of correct responses in the working memory task. They were informed about their cumulative earnings during each break. A participant could earn on average up to 15 TRY ( $~ 8$ USD) in a single temporal bisection session.

\section{Visual Discrimination Task:}

We ran temporal bisection task as it is typically reported in the literature, namely the use of left hand for short and right hand for long categorizations. However, in order to control for possible biases in the left and right hand response times, we also tested the participants on a visual discrimination task in the last session. This task required them to respond using either their left ("V" key) or right index finger ("N" key) contingent upon the location of a black circle presented either on the right or the left side of the square in a pseudo-randomized order (.01 TRY per correct response). The participants were instructed to be as fast and accurate as possible. A brief beep sound was played for correct answers and a brief buzzer sound for errors. The participants could take a break and restart testing by pressing a key after every $20^{\text {th }}$ trial.

\section{Data Analysis:}


Points of subjective equality, coefficients of variation, and response times were compared between unequal probability conditions using the Kruskal-Wallis test and, where appropriate, follow-up pair-wise comparisons were conducted using the Mann-Whitney test. Data collected from paired equal and unequal conditions were compared with Wilcoxon signed rank tests. One-way ANOVA and t-test comparisons revealed the same results in all but one of the comparisons conducted (i.e., comparison of PSEs between four different equal probability groups - used as controls for unequal probability conditions for each subject). An alpha level of .05 (two-tailed) was used for all statistical analyses. Any RT that was longer than 2s (0.63\% of trials in Experiment 1 and $0.29 \%$ trials in Experiment 2) was excluded from the RT analyses. Three participants in Experiment 2 did not attend the Visual Discrimination Task.

Optimality Analysis:

The expected gain in the temporal bisection task can be calculated as in the switch task (Balc1 et al, 2009). Although, in the switch task the participants physically switch from the location associated with short duration to the other location associated with long duration, in the case of temporal bisection task they can be considered as switching from a short categorization state to a long categorization state as time elapses (e.g., Machado \& Keen, 2003). The reward maximization here requires participants to take account of not only exogenous probabilities (i.e., $\left.p\left(T_{s}\right)\right)$ but also their own timing uncertainty (i.e., $\mathrm{CV}$ ). This relation is described in detail below.

The choice proportions gathered from the temporal bisection task can be well described as a cumulative Gaussian distribution (fit using least squares method in our 
study); the mean of the best-fit normal cumulative distribution function can be treated as the targeted point of subjective equality (PSE; criterion) and its coefficient of variation (CV) can be treated as an index of timing uncertainty. The expected gain for a given estimate of timing uncertainty $(\mathrm{CV})$, reference duration probabilities, and payoff matrix (payoffs associated with two correct and two incorrect categorizations) can be computed for different possible target PSEs. Target bisection point that maximizes the expected gain can be identified as the optimal target PSE for that given level of timing uncertainty tested with the corresponding task conditions (i.e., reference duration probabilities and payoff matrix). Here, the expected gain for a given target bisection point is the sum of the relative values of different consequences, where the relative value is the gain associated with a given consequence weighted by the probability of attaining it (Balc1 et al, 2009). Specifically, it is the payoff matrix weighted by the probability of different consequences (correctly categorizing the short reference duration as short and long reference duration as long, and incorrectly categorizing the short reference duration as long and long reference duration as short) determined jointly by the level of the participants' endogenous timing uncertainty and the probability of short and long reference durations (exogenous probabilities).

Equation 1 defines the expected gain for an estimate of the target bisection point ( $\hat{t})$ and the level of endogenous timing uncertainty $(\hat{\omega})$.

$$
\begin{aligned}
& E G(\hat{t})=g\left(\sim T_{S}\right) p\left(T_{S}\right) \Phi\left(T_{S}, \hat{t}, \hat{\omega} \hat{t}\right) \\
& +g\left(T_{S}\right) p\left(T_{S}\right)\left(1-\Phi\left(T_{S}, \hat{t}, \hat{\omega} \hat{t}\right)\right) \\
& +g\left(T_{L}\right)\left(1-p\left(T_{S}\right)\right) \Phi\left(T_{L}, \hat{t}, \hat{\omega} \hat{t}\right) \quad \text { [Equation 1] } \\
& +g\left(\sim T_{L}\right)\left(1-p\left(T_{S}\right)\right)\left(1-\Phi\left(T_{L}, \hat{t}, \hat{\omega} \hat{t}\right)\right)
\end{aligned}
$$


where $\hat{\omega}=\hat{\sigma} / \hat{t}, \hat{\sigma}$ is the standard deviation and $\hat{t}$ is the participant's target point of subjective equality (PSE) both estimated from fits to the participant's choice proportions (best fitting standard deviation and mean values). $T_{s}$ and $T_{L}$ are the short and long reference durations, $p\left(T_{s}\right)$ is the probability of the short reference duration, $g$ denotes the payoff matrix (e.g., $\mathrm{g}\left(T_{L}\right)$ is the gain associated with a correct categorization of the long reference duration, $g\left(\sim T_{S}\right)$ is the loss associated with categorizing the short duration as long). $\Phi$ denotes the normal cumulative density function with mean $\hat{t}$ and standard deviation $\hat{\omega} \hat{t}$ that can be evaluated at any arbitrary time point in the trial such as the short $\left(T_{s}\right)$ or long $\left(T_{L}\right)$ reference durations. These values can be computed for different possible $\hat{t}$ s and the optimal bisection point can be identified numerically by finding the $\hat{t}$ value that maximizes the expected gain. For instance, Figure 5 shows expected reward curves calculated according to Equation 1 for five different probability conditions and given levels of timing uncertainty. The locations of the peaks of these curves are the optimal target bisection points for the corresponding condition and the level of timing uncertainty. Note that in order to capture the scalar property (Gibbon, 1977) during these computations $\hat{o}$ is set proportional to $\hat{t}$ based on the estimate of the participant's coefficient of variation.

Figures $2 \mathrm{~A} \& 2 \mathrm{~B}$ depict the relationship between optimal temporal strategy and the level of timing uncertainty for different exogenous probability conditions on the basis of the expected gain function described above. Specifically, Figures 2A (for Experiment 1) \& $2 B$ (for Experiment 2) show the expected gains for different combinations of target PSEs and levels of timing uncertainty (forming a heat map) after normalizing them by the maximum possible expected gain for the corresponding level of timing uncertainty. The 
ridge of this normalized surface indicated by the black curve constitutes the optimal performance curve that denotes the optimal target PSEs for different levels of timing uncertainty separately for the five different probability conditions demonstrating the dependence of optimal strategies on the level of timing uncertainty in all of these conditions. Note that the optimal performance curves are identical for Experiment 1 and Experiment 2 for the same exogenous probability conditions due to the symmetrical payoff matrices. However a given level of deviation from optimality in terms of time units results in a larger loss in Experiment 2 due to the non-zero penalty for errors. Note the curved shape of the optimal performance curve even when $\mathrm{p}\left(\mathrm{T}_{\mathrm{s}}\right)=.50$. This is essentially due to the relationship between mean and standard deviation assumed by scalar property of interval timing.

\section{[FIGURE 2 ABOUT HERE]}

\section{RESULTS \\ Choice Proportions:}

Figures 3A \& 3B show the choice proportions separately for five different probability conditions along with the best-fit cumulative Gaussian distribution functions for Experiment 1 and Experiment 2, respectively. All $\mathrm{R}^{2}$ values for these fits were over .97 in both Experiment 1 and Experiment 2. The median $\mathrm{R}^{2}$ value for fits to individual participants' data was .97 for both Experiment 1 and 2. Figures 3A \& 3B show that participants modulated their choice behavior in the expected direction in response to the different experienced reference probabilities. Specifically, the psychometric function 
shifted leftward with decreasing and rightward with increasing short reference probabilities in both Experiment 1 and Experiment 2.

\section{[FIGURE 3 ABOUT HERE]}

The target PSE and the level of endogenous timing uncertainty (i.e., CV) were estimated from cumulative Gaussian fits to individual participants' choice proportions. Figures 4A \& 4B show that average empirical PSEs estimated from fits to the individual participants' data increased with increasing short reference duration probability, $F(1,3)=64.53, p<.01$, $R^{2}=.96($ Experiment 1$)$ and $F(1,3)=178.40, p<.001, R^{2}=.98$ (Experiment 2$)$. Empirical PSEs differed between equal and unequal probability conditions (within-group comparison) for all four pairs (all $p \mathbf{s}<.05)$ except for $p\left(T_{s}\right)=.25(p=.14)$ in Experiment 1 and except for $p\left(T_{s}\right)=.75(p=.60)$ in Experiment 2. When Holm-Bonferroni correction was applied, there were no significant differences. The between-group comparison of empirical PSEs between four unequal probability conditions revealed a significant overall difference, $\chi^{2}(3, \mathrm{~N}=24)=16.20, p<.01\left(\eta^{2}=.64\right)$ in Experiment 1 and $\chi^{2}(3, \mathrm{~N}=24)=16.89$, $p<.001\left(\eta^{2}=.57\right)$ in Experiment 2. The pair-wise comparisons revealed significant differences between $p\left(T_{s}\right)=.10$ vs. $p\left(T_{s}\right)=.75, p\left(T_{s}\right)=.10$ vs. $p\left(T_{s}\right)=.90$, and $p\left(T_{s}\right)=.25$ vs. $p\left(T_{s}\right)=.90$ pairs in Experiment 1 (all $\left.p \mathrm{~s}<.05\right)$ (Holm-Bonferroni corrected). The pair-wise comparisons of unequal probability conditions revealed significant differences between $p\left(T_{s}\right)=.10$ vs. $p\left(T_{s}\right)=.90, p\left(T_{s}\right)=.25$ vs. $p\left(T_{s}\right)=.75$, and $p\left(T_{s}\right)=.25$ vs. $p\left(T_{s}\right)=.90$ pair in Experiment 2 (all $p s<.05)$ (Holm-Bonferroni corrected). There were no significant differences between the four corresponding equal probability conditions neither in 
Experiment $1\left(\chi^{2}(3, \mathrm{~N}=24)=3.18, p=.37\right)$ nor in Experiment $2\left(\chi^{2}(3, \mathrm{~N}=24)=6.56, p=.09\right.$ (between-group comparison). Figures 4C \& 4D show the average empirical PSEs estimated from fits to the individual participants' data as a function of the average optimal PSEs calculated for each participant for his/her level of timing uncertainty separately for different reference probability conditions in Experiment 1 and Experiment 2 , respectively. Thin lines show the orthogonal regression fits to these data. There was a significant relationship between the empirical and optimal PSEs for both Experiment 1 slope $=.66(\mathrm{p}<.05)$ and Experiment 2 slope $=.74(\mathrm{p}<.01)$. In Experiment 1, empirical PSEs were not significantly different from the optimal PSEs (within group comparison) in any probability condition (all $p \mathrm{~s} \geq .24$ ) except for when $p\left(T_{s}\right)=.10(p<.05)$. In this condition, the empirical PSEs were significantly longer than the optimal PSEs $(p<.05)$. In Experiment 2, empirical PSEs were not significantly different from the optimal PSEs (within group comparison) in any probability condition (all $p \mathrm{~s} \geq .24$ ) except for when $p\left(T_{s}\right.$ ) $=.90(p<.05)$. In this condition, the empirical PSEs were significantly shorter than the optimal PSEs.

Finally, we calculated the AIC scores for the individual participants' choice proportions under the best fitting and the optimal cumulative Gaussian distributions for the corresponding exogenous probability conditions (using the same CV estimate). The median $\triangle \mathrm{AIC}$ scores were 9.59 and 12.96 favoring the best fitting Gaussian parameters over the optimal model parameters for Experiment 1 and 2, respectively. When the empirical choice proportions were evaluated under the best fitting and the optimal cumulative Gaussian distributions calculated for the exogenous probability condition tested in the other session, the median $\triangle \mathrm{AIC}$ scores rose to 20.61 and 22.28 for 
Experiment 1 and 2, respectively. These results suggested that the empirical behavior was closer to the optimal strategy computed for the exogenous probability conditions under which the choice behavior was actually observed.

[FIGURE 4 ABOUT HERE]

The comparison of the coefficient of variation $(\mathrm{CV})$ estimates between four different unequal probability conditions (between-group comparison) did not reveal a significant overall difference in either Experiment $1\left(\chi^{2}(3, \mathrm{~N}=24)=4.19, p=.24\right)$ or Experiment 2 $\left(\chi^{2}(3, \mathrm{~N}=24)=0.59, p=.90\right)$. The within-group comparison of $\mathrm{CV}$ estimates between unequal and the corresponding equal probability conditions did not reveal any significant differences, either (all $p \mathrm{~s}>.34)$.

\section{Expected Gains:}

We calculated the expected gain for the empirical PSEs, levels of endogenous timing uncertainty, reference probabilities, and payoff matrix and compared it to the maximum possible expected gain (MPEG) calculated separately for each participant given his/her level of timing uncertainty and the corresponding task parameters. Figures 5A \& 5B demonstrate the proportion of MPEG for possible target PSEs separately in five different reference probability conditions (calculated for average CVs) separately for Experiment 1 and Experiment 2. Visual inspection of this figure points at nearly optimal performance of our participants. The analysis of individual participant's data corroborated this observation; our participants earned $99,99.8,99.9,99$, and $99 \%$ of the 
MPEG (median) in Experiment 1, and 99.6, 97, 98, 96, and 97\% of the MPEG (median) in Experiment 2 for increasing short reference probabilities, respectively.

In a second set of analysis, we considered the gain expected from random categorization of durations as the minimum gain that can be attained; in this latter case the proportion of maximum possible expected gain was computed as (expected gainminimum gain)/(MPEG-minimum gain). The results from this second set of analysis were comparable with values gathered from the initial set of analysis: 99, 99.5, 99.7, 98, and 99\% (median) for Experiment 1 and 99.8, 98, 99, 97, and 98\% (median) of the MPEG for Experiment 2 for increasing short reference probabilities. Note that this second way of calculating the proportion of MPEGs constituted a more stringent approach for Experiment 1 whereas it was a more lenient approach for Experiment 2 (due to negative gain associated with random responding).

\section{[FIGURE 5 ABOUT HERE]}

There were no significant differences between the proportion of MPEG between equal and unequal probability conditions (within-group comparison) for neither lenient nor stringent approaches in Experiment 1 (both $p \mathrm{~s}>.17$ ) or in Experiment 2 (both $p \mathrm{~s}>.49$ ). We then computed what participants would have earned in equal reference probability condition if they adopted their unequal reference probability condition strategy (i.e., PSE), and in unequal reference probability condition if they adopted their equal reference probability condition strategy. We found that the participants would have earned significantly less if they had adopted their equal reference probability condition strategy 
in unequal reference probability conditions and vice versa, $t(23)=4.61, p<.001$ and $t(23)=3.96, p<.001$, respectively for Experiment 1 and, $t(23)=2.55, p<.05$ and $t(23)=3.63$, $p<.01$, respectively for Experiment 2 (within-group comparison). These results point at the adaptive value of the changing temporal decision strategies in response to changing probabilistic information in our task conditions.

\title{
$\underline{\text { Response Times: }}$
}

Visual inspection of Figures 6A \& 6B suggest that average RTs for short categorizations (short categorization RTs) sped up with increasing short reference probability in the absence of any apparent changes in the long categorization RTs. There was a significant relationship between short categorization RTs and short reference probability, $F(1,3)=12.71, p<.05\left(R^{2}=.85\right)$ in Experiment 1 and $F(1,3)=10.62, p<.05\left(R^{2}=.78\right)$ in Experiment 2, but this relationship did not hold for long categorization RTs, $F(1,3)=.36$, $p=.59,\left(R^{2}=.11\right)$ in Experiment 1 and $F(1,3)=2.76, p=.20,\left(R^{2}=.48\right)$ in Experiment 2.

\section{[FIGURE 6 ABOUT HERE]}

\author{
The between-group comparison of the short categorization RT-long categorization RT \\ difference score between different unequal probability conditions revealed a significant \\ overall difference, $\chi^{2}(3, \mathrm{~N}=24)=16.90, p<.001\left(\eta^{2}=.57\right)$ for Experiment 1 , and $\chi^{2}(3$, \\ $\mathrm{N}=24)=18.69, p<.001\left(\eta^{2}=.84\right)$ for Experiment 2. Pair-wise comparisons of these values \\ revealed significant differences between all unequal probability condition pairs but \\ $p\left(T_{s}\right)=.10-.25$ and $p\left(T_{s}\right)=.75-.90$ pairs $($ all $p s<.05)$ in Experiment 1 , and $p\left(T_{s}\right)=.10-.25$ pair
}


(all $p \mathrm{~s}<.05)$ in Experiment 2 (Holm-Bonferroni corrected). There were no significant differences between the corresponding equal probability conditions ( $p=.97$ for Experiment 1 and $p=.62$ for Experiment 2; between-group comparisons). Within-group pair-wise comparisons of short categorization RT-long categorization RT differences in unequal and the corresponding equal probability conditions revealed significant differences only for $p\left(T_{s}\right)=.75$ and $p\left(T_{s}\right)=.90$ conditions in Experiment 1 and for $p\left(T_{s}\right)=$ .10 and $p\left(T_{s}\right)=.25$ conditions in Experiment 2 (all $\left.p \mathbf{s}<.05\right)$. There were no significant differences left after the Holm-Bonferroni correction. The within-group comparison of the left and right hand RTs in the visual discrimination task did not reveal a significant difference, $t(23)=.43, p=.68$ for Experiment 1 , and $, t(20)=.35, p=.73$ for Experiment 2 . Thus, differences between short and long categorizations RTs cannot be accounted for simply by faster right hand responses. Short categorization RTs slowed down, whereas long categorization RTs sped with longer durations (Figures 7A and 7B) suggesting that correct categorization RTs (e.g., compare for equal probability condition) were faster than incorrect RTs. The statistical results of linear regression of short and long categorization RTs on stimulus durations are summarized in Table 1.

\section{[TABLE 1 ABOUT HERE]}

[FIGURE 7 ABOUT HERE]

\section{DISCUSSION}

We tested the temporal discrimination behavior of human participants in the temporal bisection task with different reference duration probabilities (exogenous probability) and payoffs (penalty vs. no penalty), and evaluated their performance within 
the framework of Statistical Decision Theory that incorporated the participants' endogenous timing uncertainty along with the experimentally manipulated probabilistic and reward related task parameters. Endogenous timing uncertainty was estimated from the participants' choice proportions while exogenous probabilities were manipulated by changing the reference duration frequencies. Through these analyses, the optimality of temporal bisection behavior was assessed for the first time and in its full complexity.

The participants shifted their bisection point leftward with decreasing and rightward with increasing short reference probabilities. In other words, the participants were more likely to categorize a given duration as long in the former and as short in the latter case. These findings are consistent with those reported in Jozefowiez et al, 2013, who also manipulated the relative frequency of short and long reference durations in a variant of temporal bisection task. However, the observed temporal discrimination performance was not evaluated within the framework of optimality in that study. Importantly, observed changes in temporal strategies in response to changing probabilities in our study nearly tracked optimality and significantly increased the expected gain in this task. By adjusting their choice behavior, the participants earned nearly the MPEG they could attain given their endogenous timing uncertainty level (i.e., $\mathrm{CV}$ ), experienced exogenous probability (i.e., $p\left(T_{s}\right)$ ) and the payoff matrix. Note that optimality in this task did not only depend on the exogenous probabilities but also on the level of the participants' endogenous timing uncertainty (depicted in Figure 2, see Optimality Analysis Section).

The relationships depicted in Figure 2 highlight a ubiquitous feature of temporal decision-making; time-based decisions are by default subject to timing uncertainty and its 
psychophysical properties, integral elements of human and non-human animal interval timing ability. As the participant aims at an earlier bisection point (switching from a short to a long categorization state), the absolute level of timing noise (i.e., standard deviation) that the participant is confronted with is effectively reduced. On the other hand, in our task this tendency might be counter-acted by the higher probability of short target intervals if manipulated as such in the task condition. This is why the optimal target bisection points are farther apart between the different exogenous probability conditions for higher levels of timing imprecision. Thus, temporal bisection task as utilized in this work and its optimality analysis are closely linked to the interval timing ability in its full complexity.

These findings overall suggest that humans can adjust their temporal bisection decisions in a normative fashion in response to changing task-relevant probabilistic information while taking account of the level of their own endogenous timing uncertainty. Note however that although empirical PSEs followed the optimal PSEs closely, this relationship was not perfect (see Figure 4). Specifically, the participants deviated from optimality when the optimal PSEs were very close to the short or the long reference duration. This might be due to the suboptimal tendency of humans to avoid aiming at a point outside the range of reference durations or at durations that delimit the range even when they lead to reward maximization. Subtle deviations from optimality due to similar tendencies have been previously observed in motor planning studies. For instance, Trommershäuser and colleagues reported that when the optimal target motor end-point fell outside the reward region, the participants preferred not to aim for it 
whereas their motor end-points were optimal when the optimal target was inside the reward region (Trommershäuser, Gepshtein, Maloney, Landy, \& Banks, 2005).

Alternatively, the participants in our study might have simply made the short and long choices differentially without taking account of their timing uncertainty. Figures 2 and 5 on the other hand suggest that without integrating the level of timing uncertainty into their decisions, the participants would have suffered from substantial loss of reward earned particularly in Experiment 2. Our evaluation of expected gains however showed that our participants nearly maximized their gain. The nearly optimal temporal decision performance of our participants is consistent with those of earlier studies conducted with humans and non-human animals. For instance, Balcı et al (2009) and recently Kheifets and Gallistel (2012) observed nearly optimal performance of humans and mice in the switch task that also entailed taking account of the endogenous timing uncertainty, in addition to exogenous probabilities and payoffs for reward maximization. On the other hand, distinct from the temporal bisection task where the participants indicate their temporal judgments after the cessation of the timing stimulus (as requested in this work), the switch task required the participants to indicate their temporal judgments continuously during the timing stimulus.

Despite these procedural differences, the decision processes that take place during timing stimulus and that underlie temporal bisection resemble those that underlie switching decisions. For instance, Machado and Keen (2003) found that in the bisection task pigeons moved from the location associated with short to the other location associated with long reference duration typically when the stimulus duration approached and exceeded the PSE. This pattern closely resembled the typical response pattern 
observed in the switch task (e.g., Balc1 et al, 2008). On the other hand, different from the switch task, the categorizations in the temporal bisection task should also rely on poststimulus decision processes. To that end, response times provide valuable clues regarding the contribution of prospective and retrospective components of temporal bisection performance to temporal choice behavior and the relation between them (Balc1, Simen \& deSouza, 2011b; Rodríguez-Gironés \& Kacelnik, 1998).

For instance, observed patterns of changes in short and long categorization RTs as a function of test durations (Figure 7) overall suggest that at least the post-stimulus decisions in the temporal bisection task might rely on noisy evidence accumulation (e.g., Balc1, Simen \& deSouza, 2011; Ratcliff, 1978; 2001; Ratcliff \& McKoon, 2008; Ratcliff \& Rouder, 1998). Short categorization RTs slowed down whereas long categorization RTs sped with longer test durations, namely RTs were faster for correct compared to "erroneous" categorizations. Combined with the observed choice proportions, this pattern predicts longer overall RTs for durations that are farther away from the two reference durations (or closer to the PSE). This particular pattern in turn suggests that post-stimulus rate of evidence accumulation might be determined by some distance metric between the elapsed time and the criterion set between the reference durations (e.g., Allan, 2002;

Balc1 \& Gallistel, 2006; Balcı \& Simen, under review; Wearden \& Ferrara, 1995). Within this framework, the rate of evidence accumulation would be low for test durations closer to the PSE, would be high in one direction for test durations closer to the short reference duration, and would be high in the opposite direction for test durations closer to the long reference duration. In order to address the features of these possible underlying process dynamics, we supplemented our primary analyses of choice proportions and response 
times by drift-diffusion modeling of the data from Experiment 1 (Supplemental Online Material; SoM Figures 1, 2 \& 3). As expected from a decision process that relies on a distance metric between to-be-judged duration and the bisection point, these fits have also shown that drift rate became more positive with longer test durations (given positive drift rates arbitrarily favor long threshold) regardless of the probability condition (e.g., SoM Figure 3A).

Response time patterns further implied the contribution of prospective components to the temporal bisection performance. Specifically, long categorization RTs were reliably faster than the short categorization RTs (for all probability conditions except for when $\left.p\left(T_{s}\right)=.90\right)$. Faster RTs for long categorizations were expected (at least when $\left.p\left(T_{s}\right) \leq p\left(T_{L}\right)\right)$ due to an asymmetry in the nature of short and long categorizations. If the participants compared elapsing time to a criterion also during the timing stimulus and decision processes had already favored a long categorization prior to the cessation of this stimulus, then the participants could commit to these decisions from thereon. In those cases, the participant would simply wait for the cessation of the timing stimulus to indicate the decision that had been already made during the timing stimulus (note that in our task the participants were not allowed to respond during the stimulus). On the other hand, short categorizations cannot benefit from these response time gains, as the participants should not commit to short categorizations prior to the stimulus cessation (except when $p\left(T_{s}\right)=1$ ); the current stimulus can last longer and eventually favor a long categorization. Note that if the participant had not made the long categorization during the timing stimulus, s/he would have to rely on post-stimulus evidence accumulation to 
reach a categorization decision and the frequency of these cases would decrease with increasing test duration.

Pre-commitment to long categorizations during the timing stimulus would result in a response time gain in terms of motor preparedness, which would in turn result in faster long categorization RTs compared to short categorization RTs. Based on the assumption that the participants can commit to long decisions made during the timing stimulus, we would expect non-decision delay to decrease with test duration, which was also supported by our drift-diffusion model fits (e.g., SoM Figure 3B).

The effect of reference duration probability manipulations on response times can also be evaluated from the formal perspective. Our drift-diffusion model fits suggest that the starting point moved from a point close to long threshold to an unbiased point or closer to the short decision threshold (depending on the fit type) with increasing short reference probability. Non-decision time decreased with increasing short reference probability irrespective of the fit type. When drift rate (i.e., the rate of evidence accumulation for short and long judgments) was allowed to vary between different exogenous probability conditions, it decreased and became more negative as the short reference probability increased. These results suggest that the modulation of choice behavior in temporal bisection task in response to changing reference duration probabilities might be due to the modulation of the starting point of evidence accumulation and/or the rate of evidence accumulation itself. The effect of probability manipulations on evidence accumulation can be accounted for by changes in the target criterion set between short and long references (e.g., Balcı \& Gallistel, 2006). On the other hand, we did not observe any effect of short reference probability on long 
categorization RTs. The sensitivity of short but not long categorization of response times to reference duration probabilities might be due to an advantage that benefits exclusively long decisions described above, namely commitment to long categorization decisions during stimulus. Further studies are needed to better understand the precise nature of this relationship.

The asymmetry mentioned above has been recently demonstrated also in the temporal generalization task (Klapproth \& Müller, 2008; Klapproth \& Wearden, 2012). In this task, the participants are familiarized with a standard and asked to judge if an experienced time interval is same or different from this standard interval. The resultant proportion of same judgments as a function of test durations results in a generalization gradient that peaks at the standard duration. Klapproth and colleagues tested human participants under two conditions; accuracy and speed. In the speed condition, they were asked to make their decisions as soon as possible whereas in the accuracy condition, they were allowed to take their time. Different from our study, they allowed the participants to respond prior to the cessation of the timing stimulus and measured response times starting from the onset of the timing stimulus. In both studies, they observed that under speeded conditions the response times for same judgments increased linearly with the tobe-judged time interval whereas the response times for different judgments increased until the standard duration and stayed stable from there on. These observations provide support for our claim that the participants are indeed engaged in decision making during the timing stimulus and thus they do not exclusively rely on post-stimulus decisions. Different from studies from Klapproth and colleagues, we did not allow participants to indicate their decisions prior to the cessation of the stimulus and response times were 
indexed as the time elapsed between stimulus offset and the response. It is possible that if we allowed participants to respond during the stimulus, we would have observed a similar pattern for long judgments. Further studies are needed to test this interesting prediction.

It could be argued that the participants did not pay attention to the time and simply chose the interval that was most frequent (especially in conditions with .9 probabilities). If this was the case, one would expect the choice function to be a flat line either at $\mathrm{p}($ long $)=0$ or $\mathrm{p}($ long $)=1$ depending on the probability of reference durations. A subgroup of participants indeed exhibited loss of temporal control over their choice behavior in a similar task utilized by Jozewofiez et al (2013), suggesting that those participants did not integrate temporal processing in their choice behavior. On the other hand, all of our participants exhibited a good level of temporal control over their choice behavior (even in extreme probability conditions), which entailed reliance on temporal processing along with the probabilistic information.

Another issue that relates to our findings is the possible specificity of the modulation of temporal choice behavior (by probabilistic information) to the difficult task conditions (e.g., 1:1.5 ratio of short and long reference durations with a secondary task to suppress chronometric counting in our study). On the face value such specificity might appear to challenge the generalizability of the optimal temporal risk assessment performance based on the modulation of the choice behavior. On the other hand, our formulation of the risk in temporal bisection integrates the endogenous timing noise with the exogenous probabilistic information and predicts the specificity of temporal choice 
behavior modulation (as a result of exogenous probabilities) to the difficult task conditions.

In order to demonstrate this point, we present the expected gain curves for the easy task conditions (i.e., 1:4 and 1:9 ratios) given the average $\mathrm{CV}$ values estimated and payoff matrices used in Experiment 1 and Experiment 2 (see Supplemental Material Figures $4 \& 5$ ). The visual inspection of the expected reward curves suggested that when the same risk formulation is applied to the easier task conditions, a wide range of target PSEs (rather than a single target PSE) would ensure the maximum possible expected gains. Thus, the easier task conditions with higher temporal discriminability are insensitive to the evaluation of optimal performance. This very issue also becomes apparent with the visual inspection of the expected gain surfaces for very low (psychologically implausible) CV values; when the discriminability of the durations are easy due to high temporal precision a relatively wide range of target PSEs will nearly maximize the gain. The penalty for incorrect categorizations in Experiment 2 was utilized primarily to achieve a more sensitive evaluation of optimal performance with more stringent expected reward curves.

The reward maximization based approach to temporal bisection performance also suggests a resolution to the controversy regarding the location of the PSE (Balc1 et al, 2011a). Temporal bisection task is typically utilized with equal reference duration probabilities in the literature and these studies report PSEs to be closer to the geometric mean of the reference duration in non-human animals (e.g., Church and Deluty, 1977) and closer to the arithmetic mean of the reference durations for humans (e.g., Balc1 and Gallistel, 2006). The location of the PSE has been claimed to have implications 
regarding the nature of subjective time scale (Montemayor and Balc1, 2007; Yi, 2009).

However, reward maximization approach to temporal bisection performance suggests that the location of the PSE might simply be a function of reward maximization, which is modulated due to different levels of timing uncertainty. Since humans have lower timing uncertainty compared to non-human animals, human PSEs are indeed expected to be closer to the arithmetic mean (see Figure 2 middle panel).

Reward maximization in temporal bisection task requires the associated choice behavior to be also independently sensitive to differences between the values of options in terms of associated reward magnitudes (e.g., Balcı et al, 2009). Findings from a number of earlier studies using variants of temporal bisection task with pigeons and rats confirmed this prediction (Bizo \& White, 2004; 2005; Guilhardi et al, 2007). Specifically, in these studies animals switched from the richer option (that predicted reward earlier in the trial) to the poorer option (that predicted reward later in the trial) later in the trial when options predicted reward according different VI schedules. Likewise, they switched from poorer option (that predicted reward earlier in the trial) to the richer option (that predicted reward later in the trial) earlier in the trial. Testing the effect of similar payoff manipulations in temporal bisection task and the optimality analysis of the resultant choice behavior are needed.

As mentioned earlier, our findings corroborate those reported in Jozefowiez et al (2013), who also tested the effect of reference duration probabilities on temporal bisection choice behavior. However different from Jozefowiez et al, our study extends the investigation of the effect of reference duration probabilities on temporal bisection behavior in novel directions to include 1) a reward-maximization based approach to the modulation of choice functions that incorporates psychophysical properties of interval 
timing, 2) testing temporal bisection behavior with different payoff matrices, and 3) the analysis of response time modulation in addition to choice proportions. Furthermore, our study attempted to account for the observed changes in choice behavior and response times as a whole within the drift-diffusion modeling framework (see Appendix). There are also noteworthy procedural differences between the two studies such as the lack of feedback for the categorization of reference durations, use of drastically fewer trials for testing, and use of fewer intermediate durations in Jozewofiez et al (2013). Possibly due to the combination of these minute procedural details, a non-negligible proportion of the participants in Jozewofiez et al (2013) failed to exhibit temporal control over their choice behavior.

One of the relevant issues that relate to our findings in terms of the optimal choice behavior regards the generalizability of these findings to decisions about other quantities. Although further empirical tests are needed to address this issue, it is possible that similar effects apply to other domains that are subject to well-structured representational noise characteristics. For instance, non-verbal numerosities as another magnitude-based representation are also represented with scalar variability (e.g., Platt \& Johnson, 1971; Whalen, Gallistel \& Gelman, 1999). The temporal bisection task can be utilized also with numerosities. In such a task, the judgments would take the form of closer to the "fewer" vs. "more" options. Our prediction is that in such tasks the endogenous noise characteristics along with exogenous probabilities can also be integrated in the categorization decisions about numerosities. Previous data from rats suggest that the endogenous noise might indeed be a factor that affects decisions about numerosities (Mechner, 1958). We are currently conducting experiments to address this issue with 
mice. The test of any other domain (e.g., distance) should also take account of the corresponding representational noise characteristics while evaluating the empirical performance within the framework of optimality. These would constitute good follow-up tests of our theoretical approach and its generalizability.

\section{Conclusion}

Our results showed that human participants can optimize their decisions in the temporal bisection task where reward maximizing strategies depended on both exogenous probabilities of different outcomes and the level of participants' timing uncertainty. Thus, our results suggest that human participants might have an accurate representation of these parameters and can integrate them into their decisions in a close to normative fashion. Optimal performance held for both lenient and stringent (with penalty for errors) reward functions. Briefly, this study constituted an example of optimal human temporal riskassessment under endogenous timing uncertainty and experienced exogenous probabilities, and extended it to the domain of temporal bisection. The modulation of the categorization response times furthermore suggested that temporal bisection performance might be underlain by noisy evidence accumulation processes with both prospective (i.e., during timing stimulus) and retrospective (i.e., post-stimulus) decision components (Balci et al, 2011b). Our analysis of the response times also showed that short categorization but not long categorization response times were sensitive to the manipulation of the reference duration frequencies, which might be due to the asymmetry between these decisions in terms of commitment to them before timing stimulus terminates. Future studies can 
extend the investigation of these processes to the domain of numerosity that is known to abide to similar psychophysical properties with interval timing (Gallistel \& Gelman, 2000; Meck \& Church, 1983; Meck, Church \& Gibbon, 1984; Whalen, 1999).

\section{Acknowledgments}

This work was supported by FP7 Marie Curie PIRG08-GA-2010-277015, TÜBİTAK 1001 (\#111K402) and Bilim Akademisi (BAGEP-2013) to FB. 


\section{REFERENCES}

Allan, L. G. (2002). Are the referents remembered in temporal bisection? Learning \& Motivation, 33, 10-31.

Balc1, F., Freestone, D., Gallistel, C. R. (2009). Risk assessment in man and mouse. Proceedings of the National Academy of Sciences (USA). 106, 2459-2463.

Balc1, F., Freestone, D., Simen, P., deSouza, L., Cohen, J. D., Holmes, P. (2011a). Optimal Temporal Risk Assessment. Frontiers in Integrative Neuroscience. 5, 115.

Balc1, F., Gallistel, C. R. (2006). Cross-domain transfer of quantitative discriminations: Is it all a matter of proportion? Psychonomic Bulletin \& Review. 13, 636-642.

Balc1, F., Papachristos, E. B., Gallistel, C. R., Brunner, D., Gibson, J., Shumyatsky, G. P. (2008). Interval timing in the genetically modified mouse: a simple paradigm. Genes Brain and Behavior. 7, 373-384.

Balcl, F., Simen, P., deSouza, L. (November, 2011). A drift-diffusion account of response time in retrospective temporal decision-making, 52nd Annual Meeting of the Psychonomic Society, Seattle, U.S.A.

Balc1, F., Simen, P., Niyogi, R., Saxe, A., Hughes, J., Holmes, P., Cohen, J. D. (2011b). Acquisition of decision-making criteria: reward rate ultimately beats accuracy. Attention, Perception \& Psychophysics. 73, 640-657.

Bizo, L.A., White, K.G. (1994). The behavioral theory of timing: reinforcer rate determines pacemaker rate. Journal of the Experimental Analysis of Behavior $61,308-321$.

Bizo, L.A., White, K.G. (1995). Biasing the pacemaker in the behavioral theory of timing. Journal of the Experimental Analysis of Behavior 64, 225-235.

Buhusi, C. V., Meck, W. H. (2005). What makes us tick? Functional and neural mechanisms of interval timing. Nature Reviews Neuroscience. 6, 755-765.

Church R. M., Deluty M. Z. (1977). Bisection of temporal intervals. . Journal of Experimental Psychology: Animal Behavior Processes. 3, 216-228.

Drew, M. R., Zupan, B., Cooke, A., Couvillon, P. A., Balsam, P. D. (2005). Temporal control of conditioned responding in goldfish. Journal of Experimental Psychology: Animal Behavior Processes. 31, 31-39.

Gallistel, C.R. and Gelman, R. (1992) Preverbal and verbal counting and computation. Cognition, 44, 43-74 
Gallistel, C. R., \& Gelman, R. (2000). Non-verbal numerical cognition: From reals to integers. Trends in Cognitive Sciences, 4, 2, 59-65.

Gallistel, C. R., King, A. P., Gottlieb, D., Balci, F., Papachristos, E. B., Szalecki, M., Carbone, K.S. (2007). Is matching innate? Journal of Experimental Analysis of Behavior. 87, 161-199.

Gibbon, J. (1977). Scalar expectancy theory and Weber's law in animal timing. Psychological Review. 84, 279-325.

Gibbon, J., Church, R. M. (1981). Time left: linear versus logarithmic subjective time. . Journal of Experimental Psychology: Animal Behavior Processes. 7, 87-108.

Guilhardi, P., MacInnis, M.L.M., Church, R.M., Machado, A. (2007). Shifts in the psychophysical function in rats. Behavioural Processes 75, 167-175.

Hudson, T. E., Maloney, L. T., Landy, M. S. (2008). Optimal movement timing with temporally asymmetric penalties and rewards. PLoS Biology. 4, e1000130.

Jazayeri, M., Shadlen, M. N. (2010). Temporal context calibrates interval timing. Nature Neuroscience. 13, 1020-1026.

Jozefowiez, J., Polack, C. W., Machado, A., \& Miller, R. R. (2013). Trial frequency effects in human temporal bisection: Implications for theories of timing. Behavioural Processes.

Kacelnik, A., Brunner, D. (2002). Timing and foraging: Gibbon's Scalar Expectancy theory and optimal patch exploitation. Learning and Motivation, 33, 177-195.

Kheifets, A., Gallistel, C. R. (2012). Mice take calculated risks. Proceedings of the National Academy of Sciences (USA).

Killeen, P. R., \& Fetterman, J. G. (1988). A behavioral theory of timing. Psychol. Rev., 95, 274-295.

Klapproth, F., \& Müller, M. (2008). Temporal generalization under time pressure in humans. Quarterly Journal of Experimental Psychology, 61, 588-600.

Klapproth, F., \& Wearden, J.H. (2011). Why do temporal generalization gradients change when people make decisions as quickly as possible? Quarterly Journal of Experimental Psychology, 64, 1646-1664.

Kleiner, M., Brainard, D., Pelli, D. (2007). What's new in Psychtoolbox-3? Perception, 36. ECVP Abstract Supplement. 
Machado, A., Keen, R. (2003). Temporal discrimination in a long operant chamber. Behavioral Processes. 62, 157-182.

Mechner, F. (1958). Probability relations within response sequences under ratio reinforcement. Journal of Experimental Analysis of Behavior, 1, 109-122.

Montemayor C., \& Balcı, F. (2007). Compositionality in language and arithmetic. Journal of Theoretical and Philosophical Psychology. 27, 53-72.

Platt, J. R., \& Davis, E. R. (1983). Bisection of temporal intervals by pigeons. Journal of Experimental Psychology: Animal Behavior Processes, 9, 160-170.

Platt, J. R. \& Johnson D. M. (1971). Localization of position within a homogeneous behavior chain: Effects of error contingencies. Learning and Motivation, 2, 386414.

Ratcliff, R. (1978). A theory of memory retrieval. Psychological Review, 83, 59-108.

Ratcliff, R. (2001). Diffusion and random walk processes. In N. J. Smelser \& P. B. Baltes (Eds.), International encyclopedia of the social and behavioral sciences (Vol. 6, pp. 3668-3673). Oxford: Elsevier.

Ratcliff, R., \& McKoon, G. (2008). The diffusion decision model: Theory and data for two-choice decision tasks. Neural Computation, 20, 873-922.

Ratcliff, R., \& Rouder, J. N. (1998). Modeling response times for two choice decisions. Psychological Science, 9, 347-356.

Rodríguez-Gironés, M. A., \& Kacelnik, A. (1998). Response latencies in temporal bisection: Implications for timing models. In V. De Keyser, G. d'Ydewalle, \& A. Vandierendonck (Eds.), Time and the Dynamic Control of Behavior, (pp. 51-70). Seattle: Hogrefe \& Huber.

Simen, P., Balc1, F., deSouza, L., Cohen, J. D., Holmes, P. (2011). A model of interval timing by neural integration. The Journal of Neuroscience. 31, 9238-9253.

Trommershäuser, J., Gepshtein, S., Maloney, L.T., Landy, M.S., Banks, M.S. (2005). Optimal compensation for changes in task-relevant movement variability. Journal of Neuroscience. 25, 7169-7178.

Wearden, J. H., \& Ferrara, A. (1995). Stimulus spacing effects in temporal bisection by humans. Quarterly Journal of Experimental Psychology, 48B, 289-310.

Whalen, J., Gallistel, C.R., Gelman, R. (1999) Non-verbal counting in humans: the psycho-physics of number representation. Psychological Science, 10, 130-137 
Yi, L. (2009). Do rats represent time logarithmically or linearly? Behavioral Processes. 81, 274-279. 


\section{FIGURE CAPTIONS}

Figure 1. Graphical depiction of the temporal bisection task.

Figure 2. Normalized expected gain surfaces from perpendicular view as a function of target PSE $(\hat{t})$ and the level of timing uncertainty $(\hat{\omega})$ for five different probability conditions for the payoff matrix used in Experiment 1 (left column) and Experiment 2 (right column). Expected gains for each level of timing uncertainty $(\hat{\omega})$ have been normalized by the maximum possible expected gain that could be attained with that level of timing uncertainty. This defines a ridge (indicated by the black curves) over this surface with the same level of elevation, which defines the optimal performance curve for the corresponding task conditions. Optimal performance curves defined separately for different exogenous probability conditions prescribe the reward-maximizing target PSEs for different levels of timing uncertainty under the corresponding probability condition. Shades of gray indicate the proportion of normalized maximum expected gain. Note the dependence of optimal target PSEs on reference duration probabilities (different panels) as well as on the level of timing uncertainty ( $y$-axis). The optimal performance curves are identical for Experiment 1 and Experiment 2 for the same exogenous probability conditions.

Figure 3. Choice proportions as a function of probe durations separately for five different short reference duration probabilities for Experiment 1 (left panel) and 2 (right panel). 
Curves are best-fit cumulative Gaussian distribution functions to the average choice proportions. Error bars stand for SEM.

Figure 4. A-B) Average empirical PSEs as a function of short reference duration probabilities for Experiment 1 (A) and Experiment 2 (B). Thin line is the best-fit linear regression line. C-D) Average empirical PSEs as a function of average optimal PSEs separately for different reference probability conditions for Experiment 1 (C) and Experiment 2 (D). Bold solid lines are the best-fit orthogonal regression lines. Diagonal dashed thin lines are the identity line. Error bars stand for SEM.

Figure 5. Expected gain as a function of possible target PSEs in five different probability conditions for Experiment 1 (A) and Experiment 2 (B). Possible expected gains were calculated using average empirical CV values estimated from the corresponding probability conditions and experiments. Vertical dashed lines denote the short and long reference durations. The locations of the red filled circles show the average target PSE separately for different exogenous probability conditions.

Figure 6. Average short and long categorization response times (mean response times pooled across different test durations per participant) separately for different probability conditions in Experiment 1 (A) and Experiment 2 (B). Error bars stand for SEM.

Figure 7. Average short (A\&B) and long categorization (C\&D) response times as a function of probe durations separately for five different probability conditions for 
Experiment $1(\mathrm{~A} \& \mathrm{C})$ and Experiment $2(\mathrm{~B} \& \mathrm{D})$. Lines are best-fit linear regression lines. Error bars on the graph stand for SEM. 
Table 1. The statistical results of linear regression of short and long RTs on stimulus durations (compare to Figure 7).

\begin{tabular}{|c|c|c|c|}
\hline \multicolumn{2}{|c|}{$\mathrm{F}(1,7)=$} & Experiment 1 & Experiment 2 \\
\hline $\mathrm{p}(\mathrm{Ts})=.1$ & $\begin{array}{l}\text { Short RT } \\
\text { Long RT }\end{array}$ & $\begin{array}{l}7.15, p<.05, R^{2}=.51 \\
33.60, p<.001, R^{2}=.83\end{array}$ & $\begin{array}{l}8.75, p<.05, R^{2}=.56 \\
75.62, p<.001, R^{2}=.92\end{array}$ \\
\hline $\mathrm{p}(\mathrm{Ts})=.25$ & $\begin{array}{l}\text { Short RT } \\
\text { Long RT }\end{array}$ & $\begin{array}{l}15.67, p<.01, R^{2}=.69 \\
117.77,<.001, R^{2}=.94\end{array}$ & $\begin{array}{l}1.52, p=.26, R^{2}=.18 \\
\mathbf{1 1 8 . 2 2}, p<.001, R^{2}=.94\end{array}$ \\
\hline $\mathrm{p}(\mathrm{Ts})=.5$ & $\begin{array}{l}\text { Short RT } \\
\text { Long RT }\end{array}$ & $\begin{array}{l}34.18, p<.001, R^{2}=.83 \\
77.83, p<.001, R^{2}=.92\end{array}$ & $\begin{array}{l}84.18, p<.001, R^{2}=.92 \\
95.34, p<.001, R^{2}=.93\end{array}$ \\
\hline $\mathrm{p}(\mathrm{Ts})=.75$ & $\begin{array}{l}\text { Short RT } \\
\text { Long RT }\end{array}$ & $\begin{array}{l}2.06, p=.19, R^{2}=.23 \\
\mathbf{5 5 . 8 7}, \mathbf{p}<\mathbf{. 0 0 1}, \mathbf{R}^{\mathbf{2}}=\mathbf{. 8 9}\end{array}$ & $\begin{array}{l}.06, p=.81, R^{2}=.01 \\
33.65, p<.001, R^{2}=.83\end{array}$ \\
\hline $\mathrm{p}(\mathrm{Ts})=.9$ & $\begin{array}{l}\text { Short RT } \\
\text { Long RT }\end{array}$ & $\begin{array}{l}4.86, p=.06, R^{2}=.41 \\
5.40, p=.053, R^{2}=.44\end{array}$ & $\begin{array}{l}\mathbf{7 . 2 5}, \mathbf{p}<. \mathbf{0 5}, \mathbf{R}^{2}=. \mathbf{5 1} \\
4.41, p=.07, R^{2}=.39\end{array}$ \\
\hline
\end{tabular}

Note. Significant relations are indicated by bold font and trends are indicated by italic font to ease visual inspection. 
Quarterly Journal of Experimental Psychology

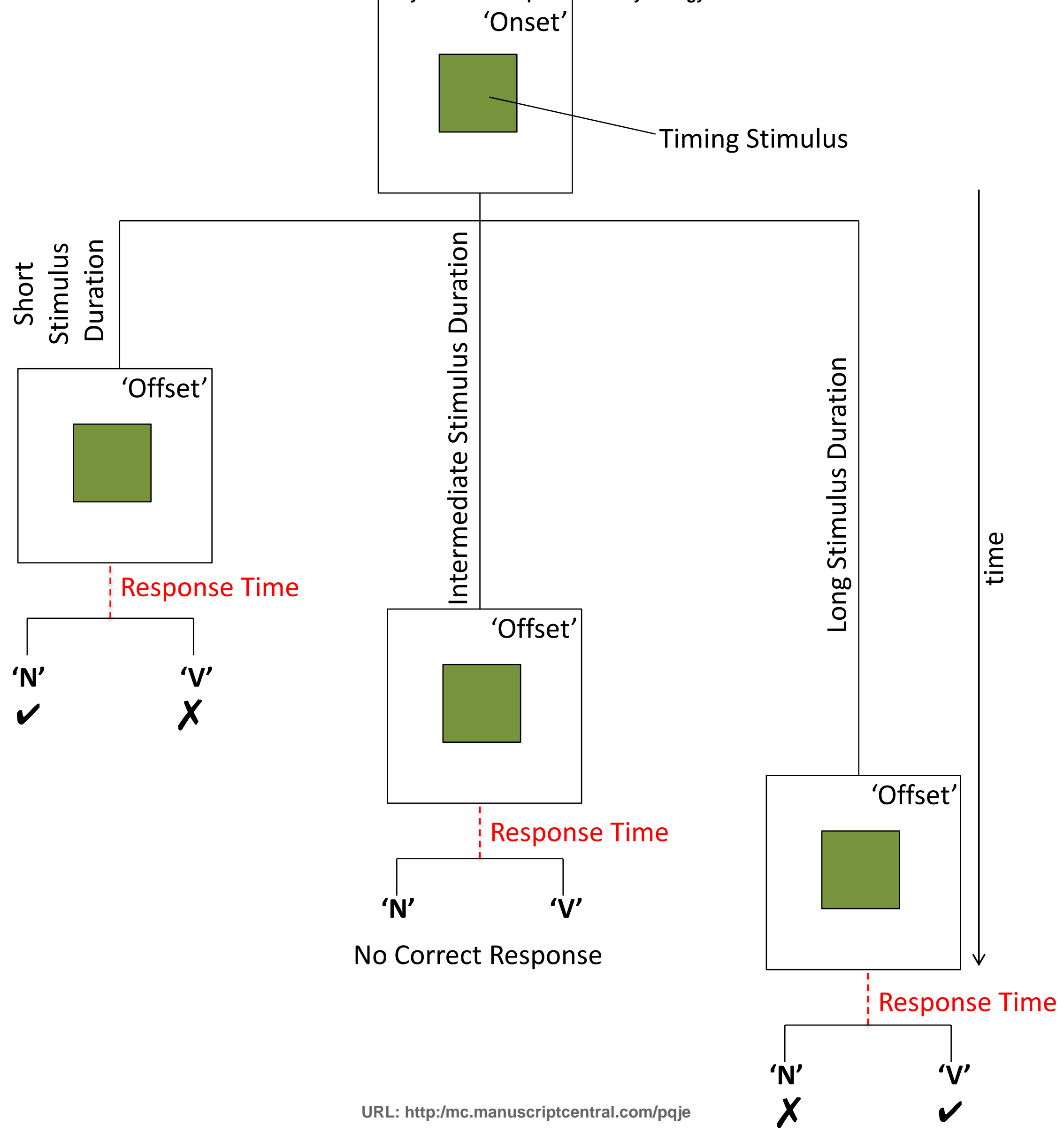


Experiment 1

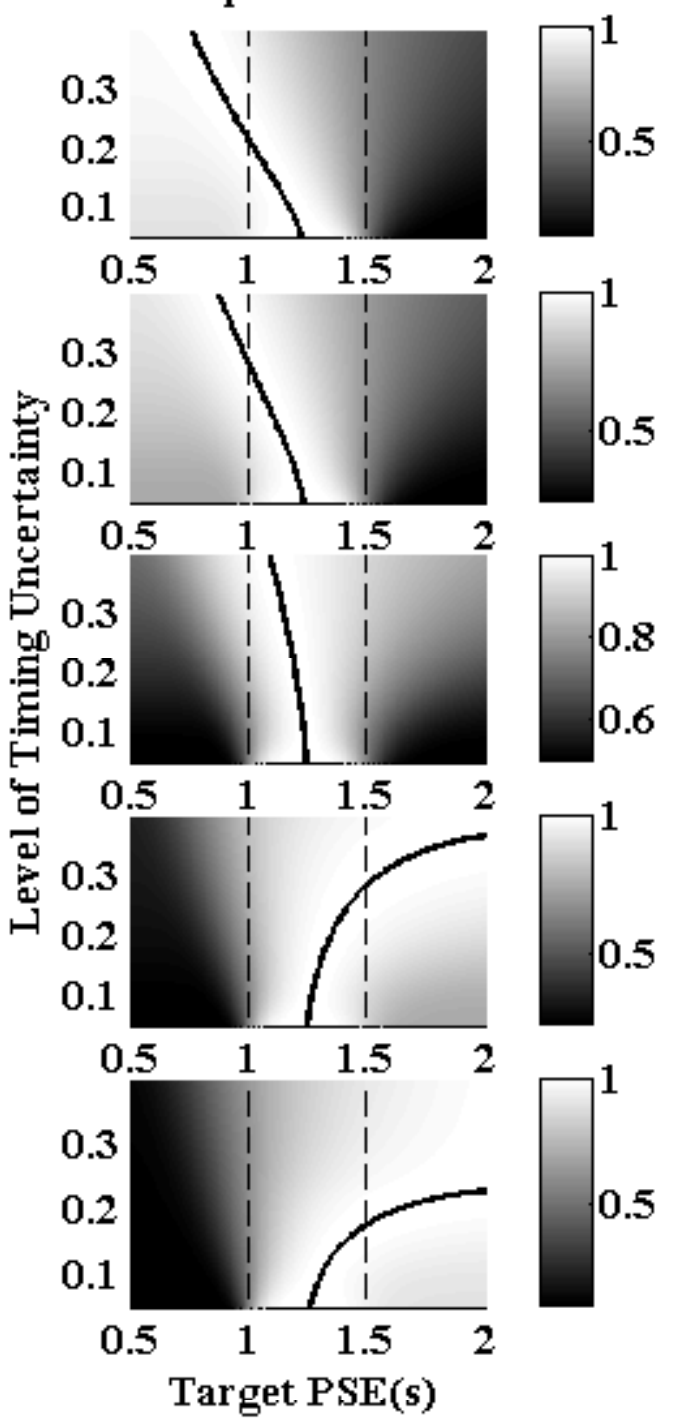

Experiment 2

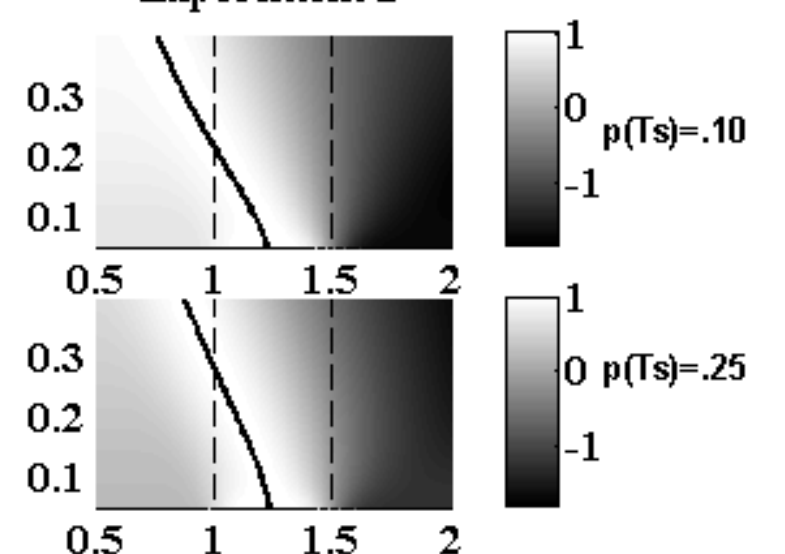

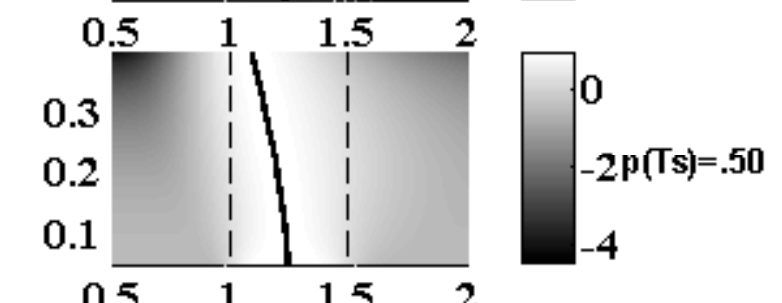
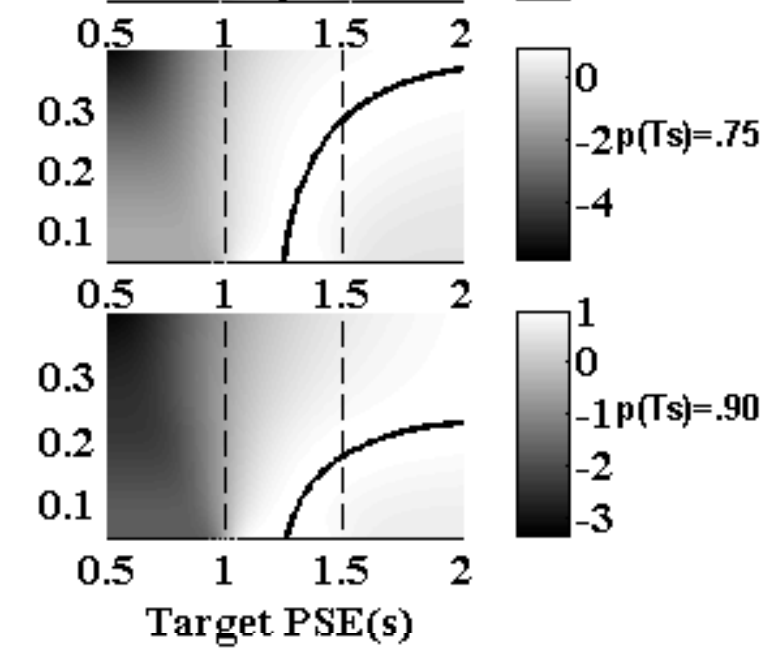

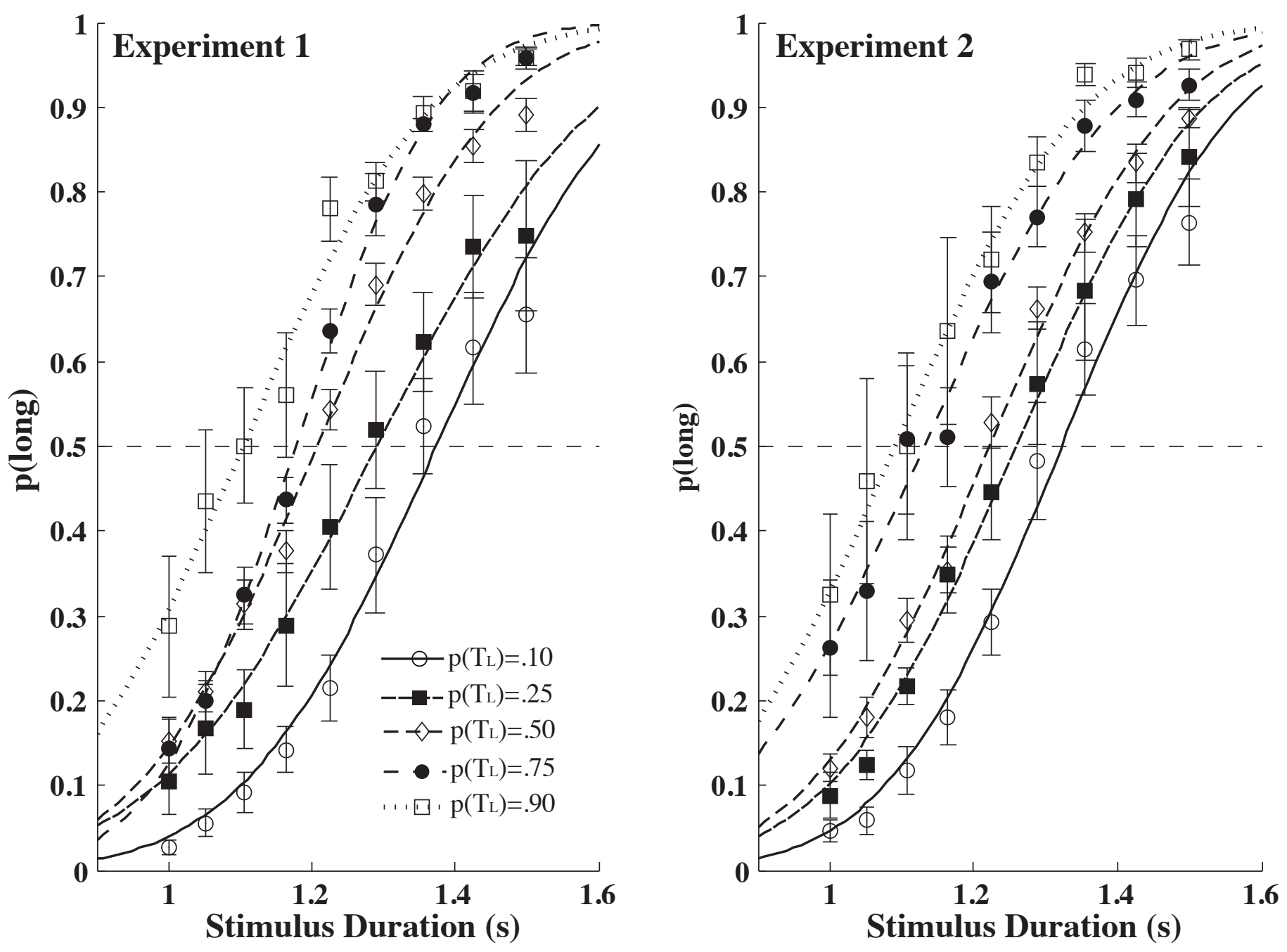

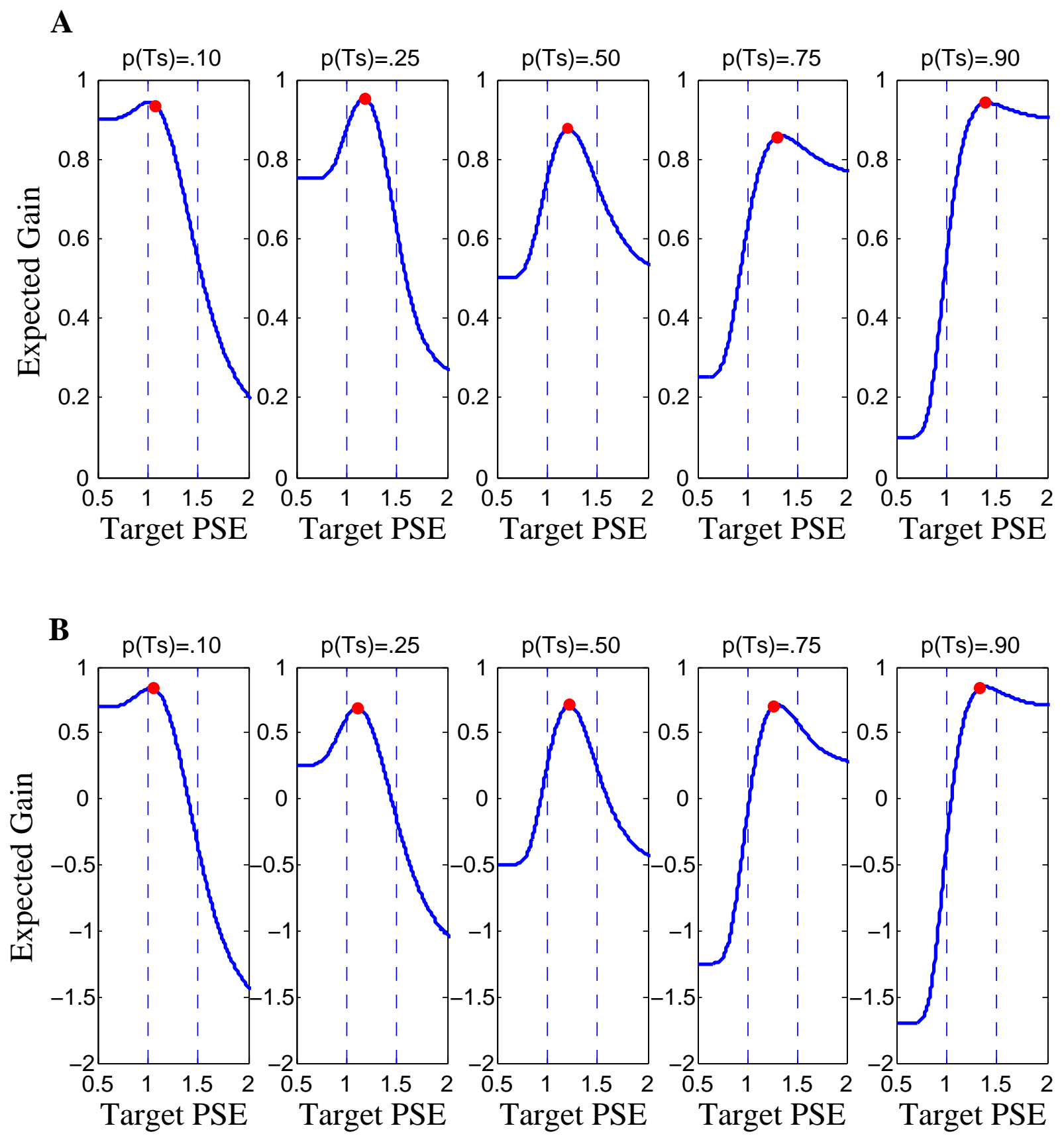

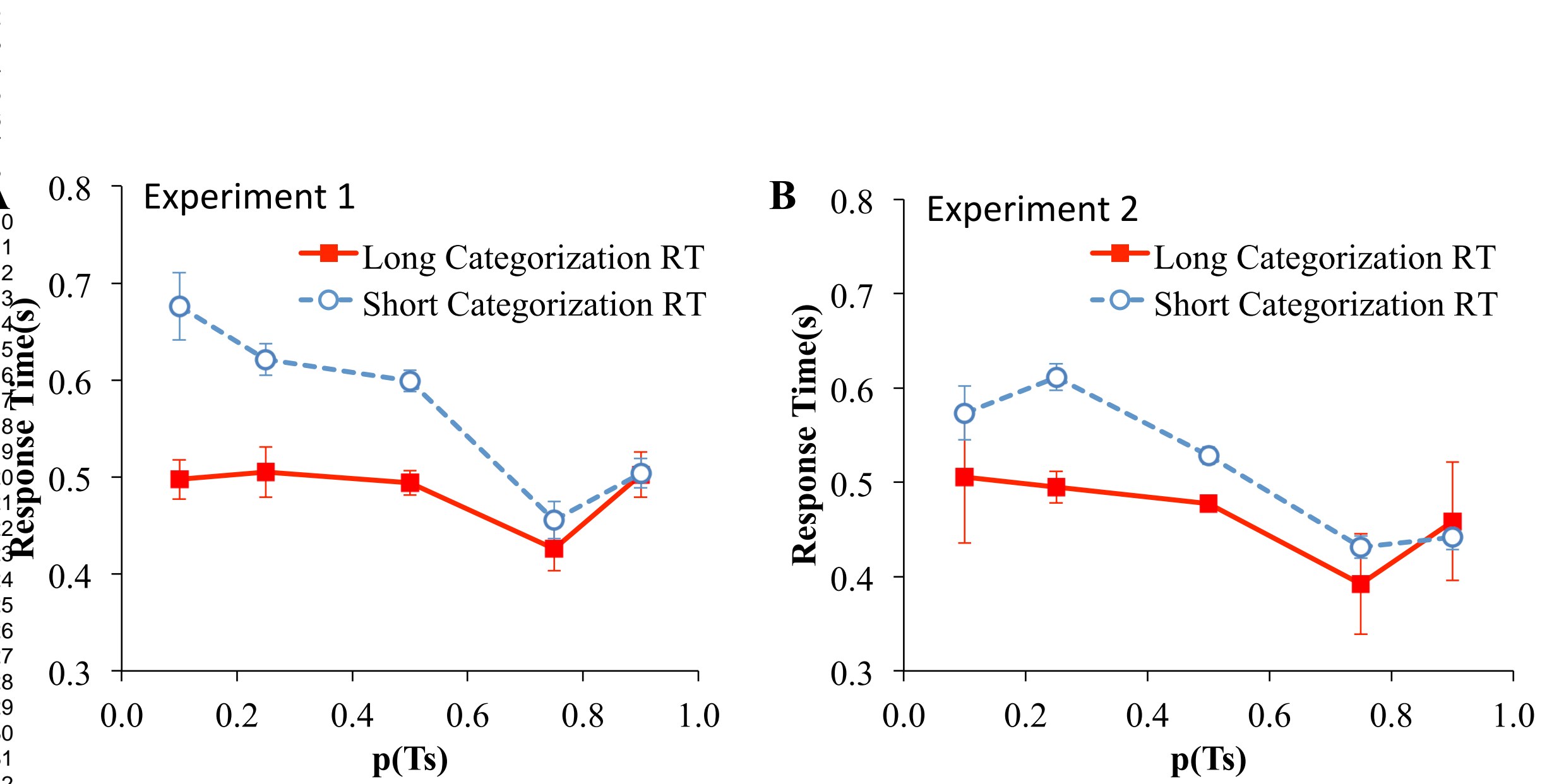


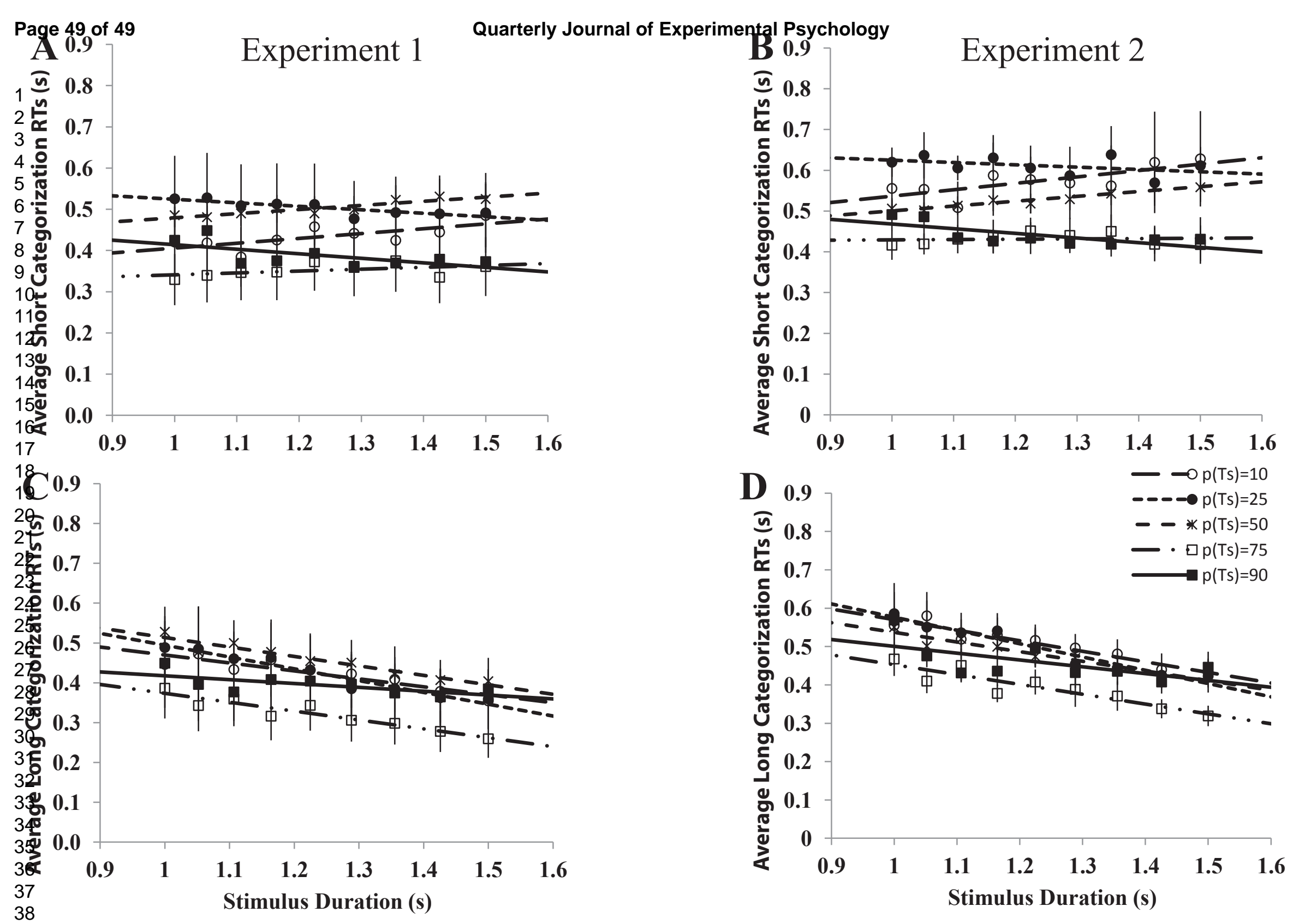

